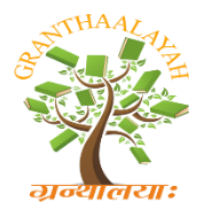

\author{
INTERNATIONAL JOURNAL OF RE
GRANTHAALAYAH \\ A knowledge Repository
}

Science

\title{
COMPARISON OF DIFFERENT PHENOYPIC TESTS FOR IDENTIFICATION OF CANDIDA ISOLATED FROM ORAL CAVITY
}

\author{
Dr. Amruta B. Hooli ${ }^{1}$, Dr. Kishore G. Bhat ${ }^{* 1}$, Mrs Preeti. Ingalgi ${ }^{1}$ \\ ${ }^{1}$ Department of Central Research Laboratory, Maratha Mandal's Nathajirao G. Halgekar \\ Institute of Dental Sciences and Research Centre, Belgavi India
}

\begin{abstract}
Background and Objectives: Oral candidiasis is one of the most common fungal infections affecting the oral mucosa. Candida genus is comprised of numerous species amongst which C.albicans is the commonest followed by C. dubliniensis. Interest has been increased towards identifying the non-albicans resistant to antifungal drugs. Various techniques are developed for species identification such as traditional, rapid, molecular and automated methods. These methods have their own advantages and limitations. Though the traditional methods are complex, time consuming, they are simple and economical as well. Hence this study was conducted to compare the different methods for presumptive species identification.

Method: A total of 50 strains were isolated from patients suffering from oral candidiasis. Samples were collected from the affected area and transferred to sterile PBS. Each sample was subjected for germ tube test, lipolytic activity, chrome agar, check for chlamydsopore formation and carbohydrate assimilation test.

Results: In the present study the phenotypic tests could provisionally identify the species. Out of 50 strains 27 isolates were identified by germ tube test, 36 strains produced lipase and positive for lipolytic activity. Chlamydospore formation provided a definitive identification of only 17 isolates. Only 7 isolates showed positive for carbohydrate assimilation tests.

Conclusion: Our study clearly showed that the phenotypic methods are useful for only presumptive identification hence the molecular methods have to be performed for definitive identification.
\end{abstract}

Keywords: Lipolytic Activity; Chrome Agar; C. Krusei; C. Gullermondi; C. Albicans; C. Tropicalis.

Cite This Article: Dr. Amruta B. Hooli, Dr. Kishore G. Bhat, Mrs Preeti. Ingalgi. (2020). "COMPARISON OF DIFFERENT PHENOYPIC TESTS FOR IDENTIFICATION OF CANDIDA ISOLATED FROM ORAL CAVITY." International Journal of Research Granthaalayah, 8(1), 32-39. https://doi.org/10.29121/granthaalayah.v8.i1.2020.240. 


\section{Introduction}

The incidence and prevalence of invasive candidal infections has increased in the recent years, especially in immunocompromised patients. Candida species are dimorphic fungi that are frequently encountered in the human oral cavity and also other body sites such as vagina and gastrointestinal tract. Even though they are considered as harmless commensal, candida species have the capability to act as opportunistic pathogens when the conditions in the oral cavity alter and become favourable for their invasion of local tissues. (1)

The genus Candida contains over 200 different species that are widely distributed in nature. Only a few of them have been recovered from human mouth in both commensal state and from the patients with oral candidiasis. (2) Among them, the most common species is C. albicans accounting for almost $80 \%$ of all isolates. Other important species include C. glabrata, C. krusei, C. tropicalis, C. gullermondi, C. kefyr and C. parapsilosis. (3)

The most common method of laboratory identification of Candida species is based on morphology and physiologic characterization of isolates. The techniques mainly include microscopy, growth on nutritionally deficient media for formation of chlamydospores, growth on selective media for production of colonies with different colour and carbohydrate fermentation and assimilation tests. (4) However, in a clinical microbiology laboratory, only small panel of tests are performed for rapid and presumptive identification of Candida species.

In the present study, an effort has been made to evaluate the relative accuracy and usefulness of tests based on phenotypic characters for proper identification of candida species isolated from the oral cavity in the laboratory.

\section{Materials and Methods}

The present study was conducted in Central Research Laboratory of Maratha Mandal's NGH Institute of Dental Sciences \& Research Centre, Belgavi after obtaining clearance from institutional ethical committee. A total of 50 Candida species isolated from patients suffering from oral candidiasis were included in the study. An informed consent was obtained before enrolling the participants for the project.

The surface of the lesion was gently scraped with a disposable sterile spatula and the material was transferred to a vial containing sterile phosphate buffered saline (pH 7.2) in the laboratory, the vial contents were mixed by vortexing and 100 microlitre was inoculated on Sabaraud's dextrose agar. The plates were incubated for 72 hours. The identity of yeasts was confirmed by staining and microscopy of the colonies from the agar surface. The isolated colonies were then subcultured to chrome agar (Hi-Media) for rapid and presumptive identification of species. The plates were incubated for 48 hours and the differentiation was done visually based upon the colours formed by different Candida species as per manufacturer's instructions. Light green, dark green, metallic blue/purple and pink coloured colonies were identified as C. albicans, C. dublinensis, C. tropicalis, C. krusei and C. glabrata respectively (Figure 1). 


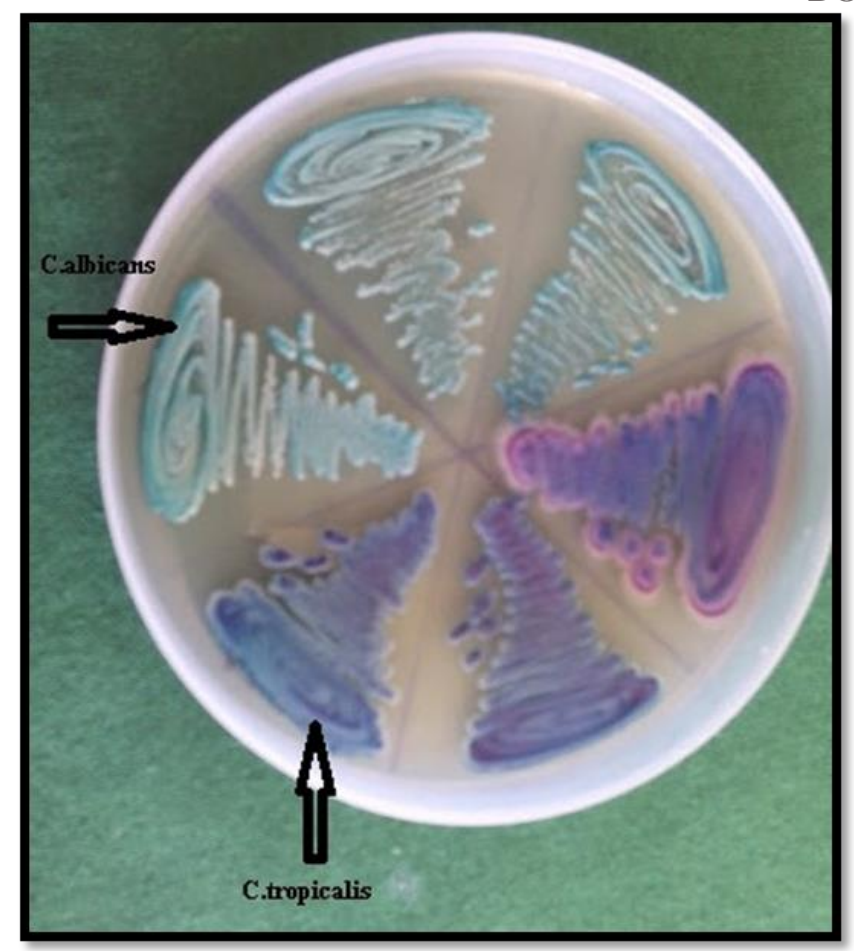

Figure 1: showing the different colour production on Chrome agar media.

Morphological identification was also done by using germ tube test for evidence of hyphal growth. This test was performed using fresh human serum and a light suspension of the subcultured colonies was inoculated. After an incubation of 2 hours, a wet mount preparation was made and examined under microscope for hyphal growth (figure 2). (5)

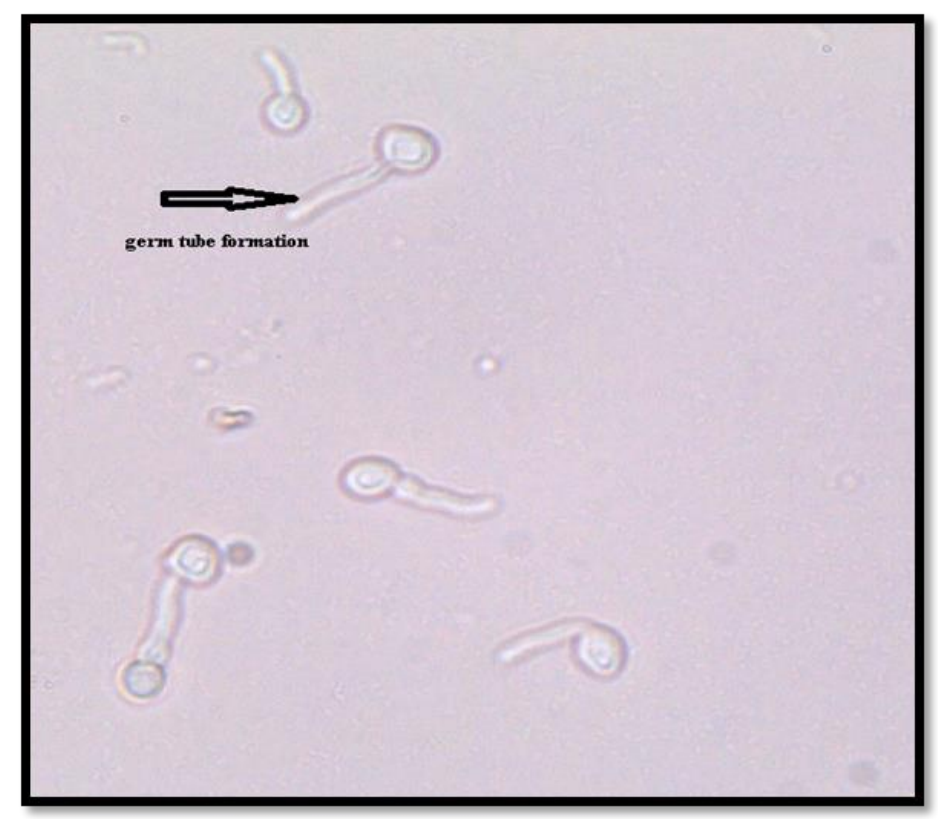

Figure 2: Germ tube formation differentiating from albicans to non-albicans. 
Further morphological study for identification of chlamydospore formation was done on corn meal agar. The isolates were inoculated in a cross hatch pattern and overlaid with a sterile coverslip, incubated for $48 \mathrm{hrs}$ and examined under microscope for typical growth pattern. (6) (Figure .3)

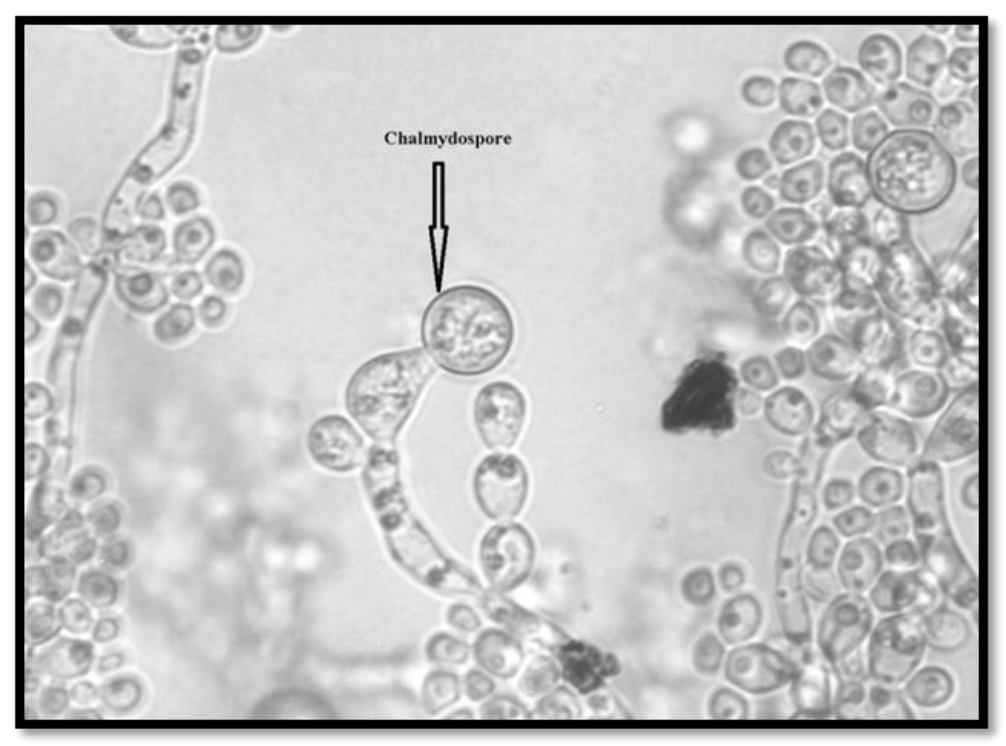

Figure 3: Chlamydopore formation on Corn meal agar

Physiological tests were performed by using modified carbohydrate assimilation test. This test utilizes a carbon and nitrogen source from yeast nitrogen base agar (YNB) with $0.002 \%$ bromocresol purple as substrate. Disks of various sugars with a concentration of $2 \%$ were placed on the surface of the agar plate and incubated for 48hour. Results were analyzed based on the circular zones of candidal growth around the carbohydrate disks. $(7,8)$ (Figure 4)

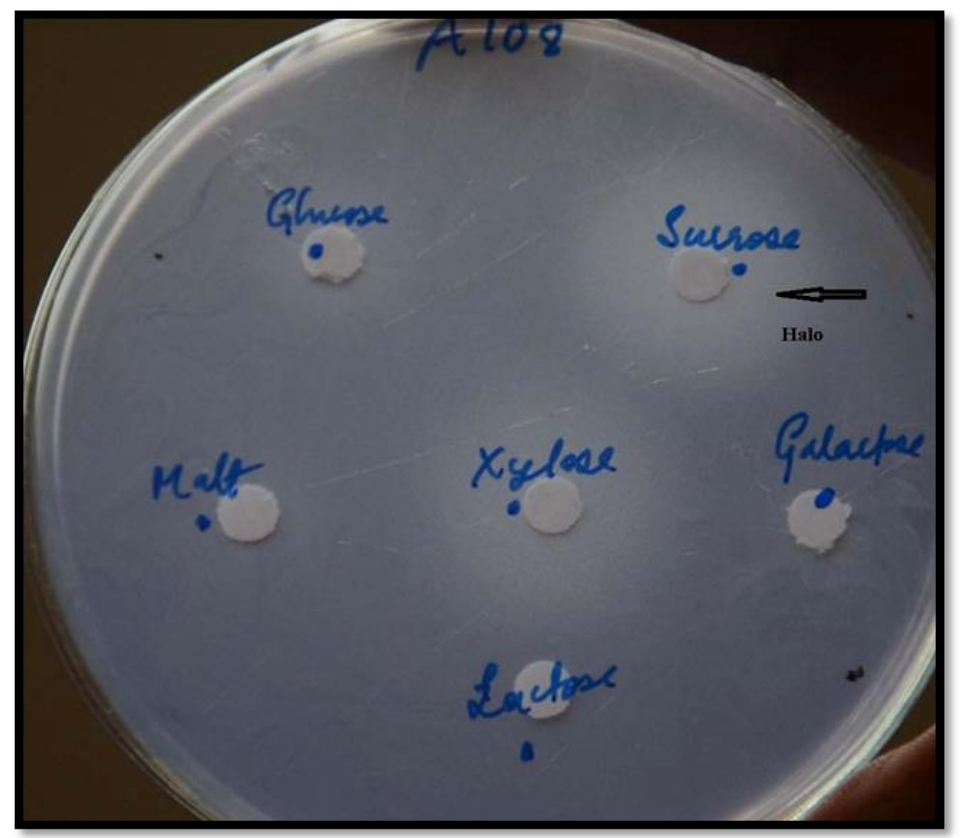

Figure 4: Showing a halo around the disk and positive for Carbohydrate assimilation test 
Additionally, the lipolytic activity of candida species was performed by using Tween-80 agar. Here the candida colonies from the primary culture were inoculated and incubated at 37-degree celsius for 48 hours. At the end of the incubation period, results were analyzed by the presence of a halo around the inoculated site observed under transmitted light was considered as positive indicating that the candida isolate tested shows possesses activity. (9)

\section{Results and Discussions}

The present study involved identification of 50 strains of candida species isolated from the oral cavity of patients with oral candidiasis using a battery of phenotypic tests. These included: germ tube test, chlamydospore formation on cornmeal agar, lipase activity on medium containing Tween-80, colour production on chrome agar and carbohydrate assimilation tests.

The results of germ tube tests showed that 27 isolates could produce hyphae and were provisionally identified as $C$. albicans. The remaining 23 strains which did not produce any hyphae were considered as species other than $C$. albicans.

Study of lipolytic activity showed that 36 strains could produce lipase, leading to break down of tween-80. They were provisionally identified as C. albicans / C. tropicalis species. Remaining 14 isolates that did not show any lipolytic activity were considered to be species other than C. albicans /C. tropicalis.

Study of chlamydospore formation pattern on corn meal agar could help in definitive identification of only 17 isolates; among them 7 were $C$. albicans, 5 were $C$. dublinensis, 1 was $C$. krusei and 2 each as belonging to $C$. tropicalis and $C$. kefyr. The remaining 33 isolates were seen to be belonging to either one of two or three different species; among them, the most common patterns were $C$. albicans / $C$. dublinensis seen in 7 isolates, followed by 6 species that could be either $C$. lusitania or C. lipolytica.

Colour production on chrome agar showed that only 13 isolates could be identified as C. albicans followed by 9 of $C$. tropicalis, 16 of $C$. dublinensis and one of $C$. glabrata. The remaining 9 isolates could not be identified conclusively as belonging to any particular species of Candida.

Finally, the carbohydrate assimilation test results showed that only 7 isolates could be definitively identified. The remaining 43 isolates were seen to belong to one or other of any two or three different species. The most common combination was C.albicans / C. dublinensis / C. catenulata that were seen in 29 isolates.

\section{Discussion}

The genus Candida is comprised of heterogeneous group of organisms and many of them are involved in causing oral mucocutaneous candidiasis and also systemic infections, especially in immunocompromised patients. (3) In recent years, an increase in the number of non-albicans Candida species resistant to antifungal drugs is recognized worldwide. Hence, correct identification of Candida species in the laboratory play an important role in designing proper antifungal therapy. (10) 
Currently, there are a number of methods for identifying Candida from clinical samples. These include traditional methods such as germ tube tests, morphological studies and carbohydrate utilization; rapid methods such as enzymatic and fluorogenic tests; commercially available and automated systems; and molecular techniques such as polymerase chain reaction (PCR). Each group of tests have their own advantages and limitations. (11) Among them, phenotypic methods even though some being complex and time consuming, are simple to perform, inexpensive and help in identification of most of the commonly occurring Candida species. Hence, we have made an attempt here to compare the different techniques and evaluate their efficacy in identification of clinical isolates of Candida from the oral cavity.

The germ tube test is considered to be a simple, economical and efficient procedure for differentiating $C$. albicans from other Candida species. (12) However, the test can not differentiate C. albicans from $C$. dublinensis. (13) In our study, when compared to chrom agar, there was discrepancy in four samples. These four isolates, identified as $C$. albicans / dublinensis by chrom agar showed negative germ tube test results. There was an overall agreement of $86.67 \%$ between the two methods.

Chlamydospre formation is a peculiar trait of $C$. albicans, $C$. dublinensis and very rarely of some isolates of $C$. tropicalis. They are dormant growth forms that arise under conditions of nutrient depletion. Even though morphology of chlamydospores in $C$. dublinensis is distinctly different from that of $C$. albicans, these are not consistent, leading to misidentification. (14) Even in our study, the agreement between chlamydospore formation with that of chrom agar results was only $68 \%$ with 16 strains showing varying results.

The ability of a particular isolate to assimilate a panel of specific carbohydrates as the sole source of carbon has been conventionally used in candidal identification. The procedure is relatively simple, inexpensive and several standard protocols are readily available. (15) Assimilation properties can be determined by two different methods: one technique is by allowing sugar solutions to diffuse from wells cut onto the agar medium that contains necessary nutrients except carbon. In the second method, growth of Candida is observed in tubes containing a basal medium with different carbohydrates after inoculation and incubation. (11) In our study, we modified the tube method protocol from earlier reports $(7,8)$ so that sugar assimilation tests could be carried out using disks loaded with individual sugars placed on a medium containing bromocresol purple. The halo created around sugar disks assimilated can be easily observed against coloured background. In the present study, except for 7 isolates, none of the Candida strains could be definitively identified as belonging to a particular species. The possibility of the presence of mixed species of Candida in the oral cavity should be ruled out as a cause for these ambiguous results.

Chrom agar Candida is a novel differential and selective medium for isolation and identification of $C$. albicans, $C$. tropicalis and $C$. krusei. The differentiation is made based on distinct colony colours produced by these species. In addition, careful observation may help detect the presence of mixed cultures of Candida species and also to presumptively identify several additional Candida species by distinct coloured halos produced by them. (16)

The Tween 80 opacity test has been proposed as a useful method for distinguishing various Candida species, based on their ability to secrete several lipolytic enzymes.(17) Occurrence of a 
halo around the inoculated site on the tween medium, when viewed with transmitted light indicates the lipolytic activity of Candida species(18)According to Slifskin (9), all strains of C. albicans and C. tropicalis should show lipolytic activity in 2-3 days and C. guillermondii and C. rogosa after 810 days of incubation. In the present study, there was no correlation between the Tween 80 test with that of any other method and non-albicans, non-tropicalis Candida strains identified by other phenotypic methods also showed lipolytic activity.

Conventional methodology has been used since a long time for identification of Candida species. Some of these methods are laborious and time consuming and require support of additional tests for correct identification of Candida species. In our study, we could see that germ tube test and chrom agar had excellent correlation and was very good in presumptive identification of select Candida species. The chlamydospre formation and Tween 80 opacity tests were not satisfactory for initial differentiation and need additional tests for confirmation. Carbohydrate assimilation tests could not definitively identify most of the candida isolates. Similar results were shown by other workers ${ }^{8}$ and possibility of mixed infections with several candida species simultaneously should also be taken into consideration before interpreting the results.

\section{Conclusions and Recommendations}

Our study very clearly shows that phenotypic methods are useful for only presumptive identification of Candida and molecular techniques such as PCR will have to be performed for definitive identification of the species.

\section{References}

[1] Marsh PD, Marlin MV. Oral fungal infections in Oral Microbiology 5th Ed. 2009; 166-179. Churchill Livingstone

[2] Akpan A, Morgan R. Oral candidiasis. Postgrad Med J. 2002; 76: 455-459.

[3] Sardi JCO, Scorzoni L, Bernardi T, Fusco-Almeida AM, Mendes Giannini MJS. Candida species: current epidemiology, pathogenicity, biofilm formation, natural antifungal products and new therapeutic options. J Med Microbiol. 2013; 62: 10-24.

[4] Williams DW, Lewis MA. Isolation and identification of Candida from oral cavity. Oral Dis 2000; 6:3-11.

[5] Campbell CK, Holmes AD, Davey KG, Szekely A, Warnock DW. Comparison of a new chromogenic agar with germ tube method for presumptive identification of candida albicans. Eur J Clin Microbiol Infect Dis 1998; 17: 367-368.

[6] 6.Larone DH. Medically important fungi: A guide to identification. 4th Ed

[7] Qadri Hussain SM, Nichols CW. Tube carbohydrate assimilation method for rapid identification of yeasts. Med Microbiol Immunol 1978; 165: 19-27.

[8] Marinho SA, Teixeira AB, Santos OS, Cuzanova RF, Ferriera CAS, Cherubini K etal. identification of candida spp. by phenotypic tests and PCR. Braz J Microbiol. 2010; 41: 286-294.

[9] Slifkin M. Tween 80 opacity test responses of various candida species. J Clin Microbiol 2000;38: 4626-4628.

[10] Ingham, C. J., Boonstra, S., Levels, S., de Lange, M., Meis, J. F. Schneeberger, P. M. (2012). Rapid susceptibility testing and microcolony analysis of Candida spp. cultured and imaged on porous aluminum oxide. PLoS ONE 7, e33818. 
[11] Neppelenbroek KH, Seo RS, Urban VM, Silva S, Dovigo LN, Jorge JH et al., Identification of Candida species in the clinical laboratory: a review of conventional, commercial and molecular techniques. Oral Dis 2014; 20: 329-344.

[12] Hoppe JE, Frey P.Evaluation of six commercial tests and the germ tube test for presumptive identification of Candida albicans. Eur J Clin Microbiol Infect Dis 1999; 18: 188-191.

[13] Campbell, C.K.; Holmes, A.D.; Davey, K.G.; Szekely, A.; Warnock,D.W. (1998). Comparison of a new chromogenic agar with the germ tube method for presumptive identification of Candida albicans. Eur J Clin Microbiol Infec Dis 17: 367-8.

[14] Kim D, Shin WS, Lee KH, Kim K, Young Park J, Koh CM (2002). Rapid differentiation of Candida albicans from other Candida species using its unique germ tube formation at 39 degrees $\mathrm{C}$. Yeast 19: $957-962$

[15] Latouche GN, Daniel HM, Lee OC, Mitchell TG, Sorrell TC, Meyer W (1997). Comparison of use of phenotypic and genotypic characteristics for identification of species of the anamorph genus Candida and related teleomorph yeast species. J Clin Microbiol 35: 3171-3180.

[16] Odds FC, Bernaerts R. CHROMagar Candida, a new differential isolation medium for presumptive identification of clinically important Candida species. J Clin Microbiol. 1994; 32: 1923-1929.

[17] Slifkin M (2000). Tween 80 opacity test responses of various Candida species. J Clin Microbiol 38: 4626-4628.

[18] Dostal J, Hamal P, Pavlickova L et al (2003). Simple method for screening Candida species isolates for the presence of secreted proteinases: a tool for the prediction of successful inhibitory treatment. J Clin Microbiol 41: 712-716.

\footnotetext{
*Corresponding author.

E-mail address: drkgbhat@ yahoo.com
} 